\title{
LASER MICROSCLEROSTOMY FOR PRIMARY OPEN ANGLE GLAUCOMA: A REVIEW OF LASER MECHANISMS AND DELIVERY SYSTEMS
}

\author{
B. D. S. ALLAN, P. P. VAN SAARLOOS, R. L. COOPER, I. J. CONSTABLE \\ Perth, Australia
}

\begin{abstract}
SUMMARY
A number of different lasers and delivery systems are currently used in experimental sclerostomy procedures. These are discussed with reference to their performance in terms of cutting accuracy and trauma to the adjacent tissues. Lasers emitting wavelengths close to the absorption peaks of water in the mid-infrared region and organic polymers in the far ultraviolet region have a short absorption pathlength in the sclera and produce the least adjacent thermal tissue trauma. These ablating lasers cannot be delivered gonioscopically, and contact endoscopic techniques trap hot expanding gases within the forming sclerostomy channel causing secondary thermal and mechanical damage. Optimal results should be obtained using an ablating laser delivered either through an open mask or a modified endoscopic system incorporating an adequate exhaust mechanism.
\end{abstract}

With improved accuracy in disc imaging and visual field analysis together with other, perhaps more subtle, indices of optic nerve fibre loss, a definitive diagnosis of primary open angle glaucoma can be made at an increasingly early stage.

An adequate reduction of the intraocular pressure in glaucomatous eyes may arrest the progression of, ${ }^{1}$ and even partially reverse, ${ }^{2,3}$ visual field loss. This can be achieved by using topical medication or fistularising surgery. The conventional practice has been to resort to surgery only when medical management proves inadequate. However, recent evidence suggests that an initial trial of topical medication may be less effective than early surgical treatment in controlling visual field loss, ${ }^{4}$ and that long-term topical medication may have a directly deleterious effect on the superficial ocular tissues and their subsequent capacity to form a functional drainage bleb. ${ }^{5}$ Despite this, primary surgical treatment is not routinely offered at present because of the complications associated with surgery and the greater initial demand this would place on hospital resources.

Correspondence to: Bruce Allan, The Lions Eye Institute, 2nd floor, 2 Verdun Street, Nedlands, Western Australia, Australia 6019.
Conventional surgery has two essential areas of poor control from which most of the associated complications emanate: variability of fistula size-with excessive or insufficient outflow in a proportion of cases; and trauma to the adjacent tissues - stimulating inflammation, wound healing, and fistula closure. Laser sclerostomy procedures that might be performed quickly and easily in an outpatient setting are currently being developed. Examples of lasers and delivery systems employed in sclerostomy procedures are evaluated below with reference to their possible impact in these two key areas: cutting accuracy and tissue trauma.

\section{LASERS USED IN SCLEROSTOMY PROCEDURES}

In simplistic terms, it is possible to burn, blast, or etch a channel through the sclera; and this loosely describes the three principal modes of tissue interaction by which lasers employed in sclerostomy procedures may be classified.

Lasers traumatise the surrounding tissues thermally or mechanically to a variable degree depending on the laser used. Cutting accuracy varies with the extent of adjacent tissue trauma, since the transition from the undamaged to the damaged state alters the dimensions of the tissues involved. Hence, when evaluating the lasers themselves, as opposed to delivery systems used in laser sclerostomy procedures, cutting accuracy and tissue trauma do not require independent consideration.

The way in which laser light reacts with the tissues depends essentially upon the power (energy/time) delivered to the target volume, or the power density achieved. Laser light may be considered as a stream of identical photons, or discrete packets of electromagnetic energy. The power density thus varies with photon energy (inversely proportional to wave length) irradiance (the number of photons delivered to the target area per unit time) and absorption pathlength (the distance beneath the target surface in which photons are likely to be absorbed). Other factors, in particular the temporal pulse energy pro- 
file-that is, the pattern of instantaneous changes in irradiance during a pulse-are also influential. But the essence of what occurs when laser light strikes the tissues can be grasped by consideration of photon ener ${ }_{0}^{y}$, irradiance and absorption alone.

For any laser aimed at the sclera, photon energy and absorption characteristics are normally fixed, and the power density is modulated by alterations in irradiancewhich has two important component elements: fluence per pulse and pulse length (Fig. 1). Increasing laser energy and decreasing spot size increases fluence, or the number of photons delivered to the target area. These may be released in a steady stream (continuous wave), or in a pulse. As with intravenous drug administration, a greater instantaneous concentration is achieved by bolus injection than by slow infusion. Hence a shorter pulse duration produres a greater irradiance, and a greater power density.

To impart their energy to the target tissues, photons must be absorbed rather than reflected, scattered, or transmitted. At irradiances lower than a certain threshold (that at which ionisation, absorption by free electrons, and plasma formation begins), the absorption characteristics of a tissue depend on its molecular composition, each molecule having its own wavelength dependent absorption profile. Water, for example, absorbs poorly in the visible spectrum, and is thus transparent, but has absorption peaks in the mid-infrared region. The organic scleral constituents however absorb most strongly in the far ultraviolet spectrum. Photons at wavelengths close to these absorption peaks will be absorbed within a smaller pathlength

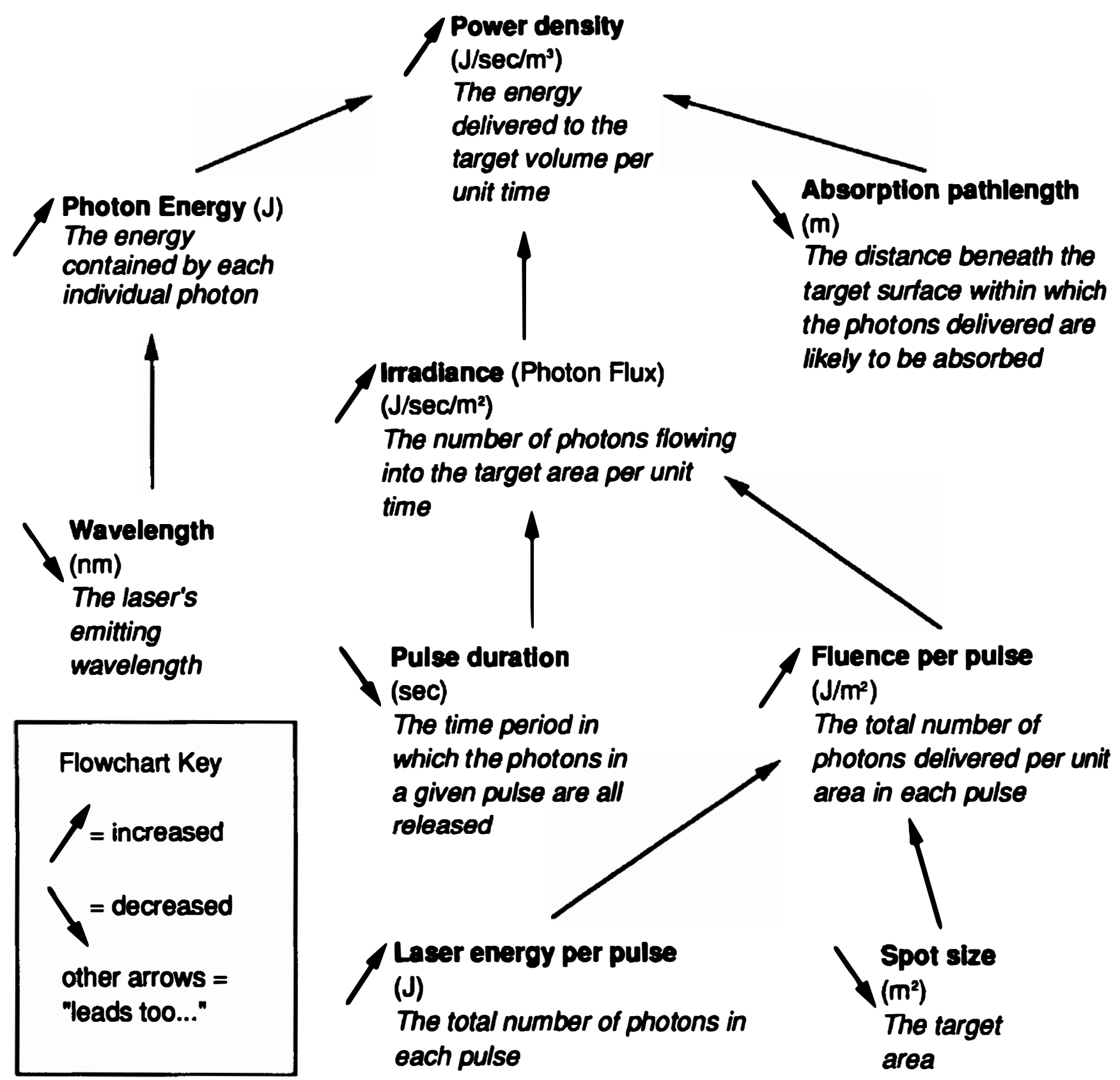

Fig. 1. A flowchart illustrating the relationship of some factors influencing the mode of laser/tissue interaction. 
beneath the target surface, or a smaller tissue volume, again producing a greater power density.

For all lasers used in sclerostomy formation, the effect of increasing power density is initially to raise the temperature of the target tissues at a rate directly proportional to the power density delivered. As irradiance, and hence power density, is increased, this linear relationship is eventually interrupted by plasma formation. Lasers at any wavelength will initiate plasma formation if irradiance is high enough. For the ultraviolet lasers whose individual photon energy is sufficient to break intramolecular bonds directly however, an additional stage between thermal interaction and plasma formation emerges as irradiance is increased. This is photodissociation.

Laser sclerostomy formation essentially requires that the organic polymers in the target tissues are fragmented and ejected in a gaseous state. This phase change can be brought about by thermal molecular fragmentationphotovaporisation; ionisation, plasma expansion, and predominantly mechanical molecular fragmentationphotodisruption; or direct, non-thermal molecular decomposition-photodissociation. Lasers producing each of these three basic modes of tissue interaction in sclerostomy formation are discussed below.

\section{PHOTOVAPORISING (THERMAL) LASERS}

Visible and infrared lasers at microsecond and longer pulse durations are predominantly thermal. All lasers currently used for retinal treatment are in this class, ${ }^{6}$ and several thermal lasers have been employed in experimental sclerostomy procedures. Examples include carbon dioxide lasers, ${ }^{7}$ argon lasers, ${ }^{8-12}$ continuous wave $\mathrm{Nd}: \mathrm{YAG}^{13-15}$ microsecond pulsed dye lasers at $666 \mathrm{~nm},{ }^{16-20}$ and mid-infrared lasers. ${ }^{21-26}$

These lasers form a sclerostomy by inducing a sudden focal temperature rise in the target tissues. Scleral collagen and glycosaminoglycans are denatured (photocoagulation). The tissue directly beneath the laser spot fragments and is ejected along with vapourised water (photovaporisation), and adjacent denatured tissue contracts. Charring (carbon deposition where oxygen has been available for burning) is seen at the wound edges. Thus thermal lasers might be thought of as melting or burning their way through the sclera.

The principle disadvantage shared by most thermal lasers is that a relatively large zone of thermal damage surrounding the sclerostomy is inevitable. Jaffe et al. ${ }^{8}$ using a high powered $(5-15 \mathrm{~W})$ argon blue-green laser with a 0.1 second pulse duration in rabbits noted a $250 \mu \mathrm{m}$ thermal damage zone about a $400 \mu \mathrm{m}$ sclerostomy. Clearly, this volume of necrotic tissue represents a significant stimulus to wound healing - macrophage and fibroblast infiltration of the damage zone was noted on day two, and drainage ceased in all fistulas by day four. This order of brevity is not unusual in rabbit studies of fistula durability; but these results are certainly no better than those obtained with conventional drainage procedures. ${ }^{27}$ Sufficient coagulative thermal damage to surrounding tissues can produce simultaneous cautery however, ${ }^{8,14}$; and since blood contains many potent fibroblast chemotactic factors, haemostasis may be particularly important in this context.

There are three principal mechanisms of collateral thermal damage in laser sclerostomy formation (Fig. 2). For the laser itself, by far the most important determinant of the extent of surrounding thermal damage is the absorption pathlength. The holmium and erbium lasers emitting wavelengths ( $2.01 \mu \mathrm{m}$ and $2.94 \mu \mathrm{m}$ respectively) close to the absorption peaks of water in the mid infrared region have a short absorption pathlength in the sclera (less than $25 \mu \mathrm{m}){ }^{6}$ This is considerably shorter than that of the other thermal lasers. As a consequence, thermal damage zones are greatly reduced.

Thermal damage zones as low as $17 \mu \mathrm{m}$ have been observed for holmium and erbium lasers in experimental conditions, ${ }^{24}$ although damage zones of up to $140 \mu \mathrm{m}^{22}$ have also been reported for the erbium laser. Details of sclerostomy size were not given. The extent of thermal damage with the erbium laser appears to vary with fluence ${ }^{24}$ and the mode of delivery. ${ }^{22}$ Another mid-infrared laser, the thulium laser, requires less energy and fewer pulses to form a sclerostomy than the holmium laser, ${ }^{25}$ but any associated improvement in the extent of thermal damage has not been reported.

To improve the performance of visible and near infrared thermal lasers, shorter pulse durations and 'dye-enhancement' have been introduced. Thermal lasers emitting outside the mid-infrared region, the dye laser at $666 \mathrm{~nm}$ and the argon blue-green laser for example, are relatively poorly absorbed by the sclera. Impregnation with a dye whose absorption peak corresponds approximately to the wavelength of the incident laser light effectively shortens the absorption pathlength, with a commensurate reduction in the surrounding thermal damage zone.

Variations on the theme of dye-enhanced absorption have included intrascleral injection of indian ink ${ }^{28}$ or silver oxide,$^{29}$ and iontophoretic impregnation with methylene blue. ${ }^{17}$ Iontophoresis, being less traumatic, is the preferable technique, although it adds up to five minutes to the procedure time. Latina et al. ${ }^{17}$ using a pulsed dye laser at $666 \mathrm{~nm}$ to perforate strips of isolated human sclera iontophoretically impregnated with methylene blue noted a thermal damage zone of $25-50 \mu \mathrm{m}$ with 1.5 and $20 \mu \mathrm{sec}$ pulse durations. Thermal damage zones were larger in rabbit studies however (up to $200 \mu \mathrm{m}$ ). Also, current iontophoresis techniques are unreliable, and the laser will not cut through sclera that is not dyed. If sclera in the target zone is not impregnated with dye throughout its full thickness, sclerostomy formation will remain incomplete. Early results from human trials underline this difficulty. Sclerostomies were incomplete in five out of twelve eyes in one trial using this technique, ${ }^{19}$ and in six of fifteen eyes in a second. ${ }^{20}$

Improvements in the thermal damage zone are gained at the expense of cauterising efficiency. Latina $e t$ al. noted bleeding as a complication using dye-enhanced absorption and the same (1.5 and $20 \mu \mathrm{sec})$ shorter pulse lengths 

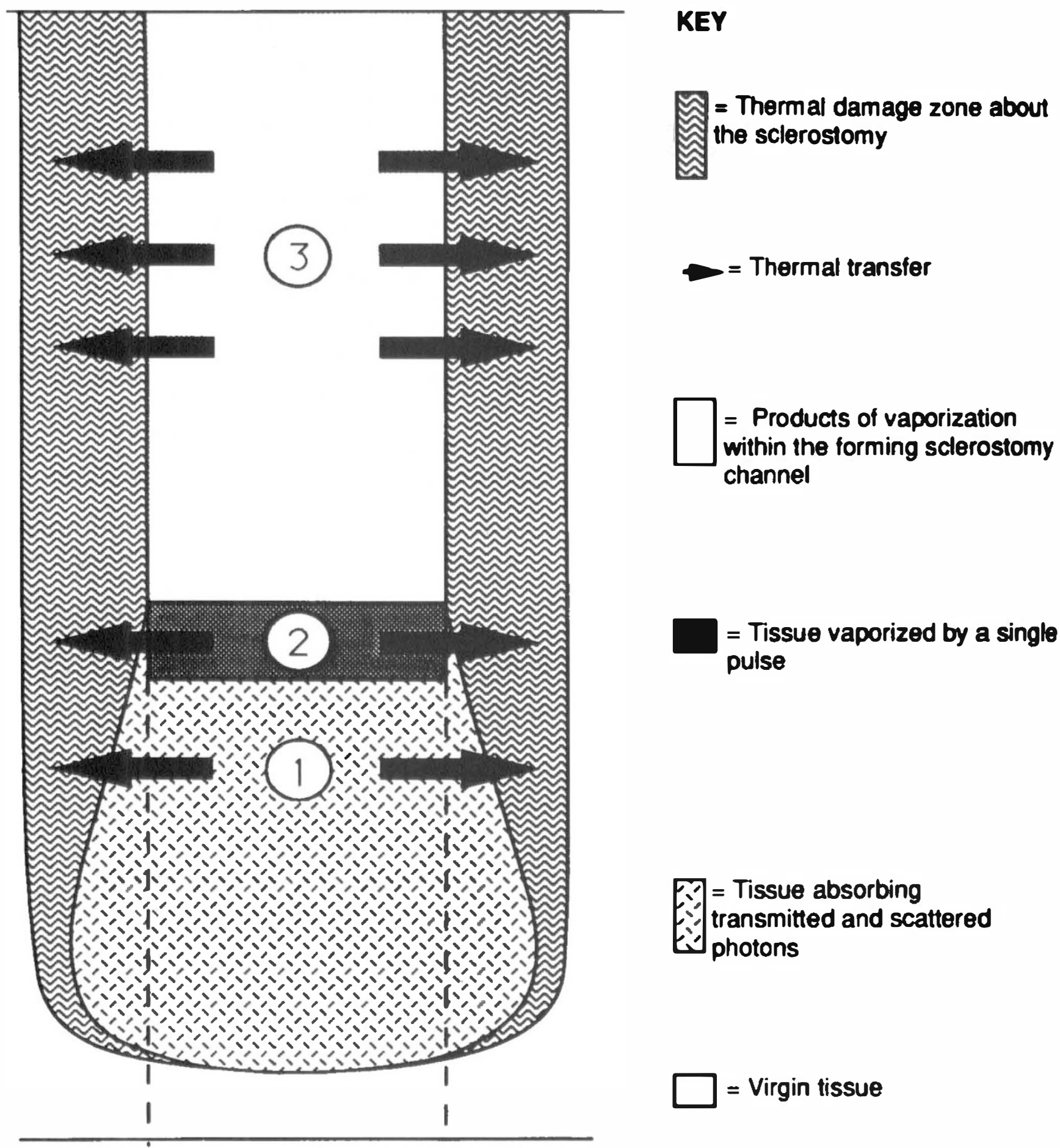
channel

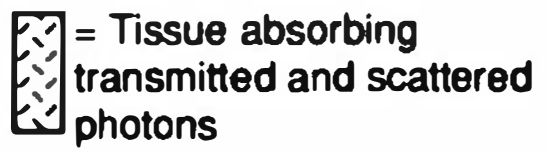

Fig. 2. A schematic illustration of the three principal mechanisms of thermal damage to the adjacent tissues in laser sclerostomy formation: 1. Direct absortion of, and lateral conduction from, transmitted and scattered photons. This increases with absorption pathlength, since poor absorption confines less photons within the portion of target tissue vaporised by a single pulse, allowing more transmission and scattering; 2 . Transfer of heat from the portion of tissue removed by a single pulse in the period between laser impact and vaporisation. This varies with the power density (proportional to fluence per pulse, pulse length, and absorption pathlength) achieved in the target tissue. Note that, again, absorption is of paramount importance, since increasing fluence also increases the number of photons available for transmission and scattering (mechanism 1), and the interval between laser impact and the commencement of vaporisation may be considerably shorter than the pulse length for well absorbed lasers (e.g. mid-infrared); 3. Transfer of heat from the products of vaporisation. This depends upon the extent to which trapping by the delivery system delays their exit from the sclerostomy. 
in their rabbit studies. ${ }^{17}$ They also comment on the trade off between decreasing thermal damage and increasing pressure wave effects at shorter pulse durations. ${ }^{17}$ The volume expansion associated with water vapourisation occurs more rapidly with a shorter pulse duration. This rapid expansion causes the target tissue to recoil, and energy is conducted away in a pressure wave. Some shuddering of the iris was observed in Latina's rabbit experiments. But at microsecond pulse durations it is unlikely that mechanical damage of this nature would be clinically significant.

\section{PHOTODISRUPTING (PLASMA FORMING) LASERS}

Q-switched Nd:YAG lasers $(1.06 \mu \mathrm{m})$ with nanosecond pulse durations are brought to a point focus where irradiances of immense proportions are achieved. Any material in the minute volume at the laser's focus is ionised and a plasma, the so-called 'fourth state of matter' in which atoms are stripped of their electrons, is formed. ${ }^{30}$ Plasma formation engenders an extremely rapid volume expansion, discharging a shock wave and significant thermal energy to the adjacent tissues. A shock wave is a supersonic pressure wave, which may be conducted through the eye with little loss of energy until a change of density is encountered at a tissue interface, between the vitreous and the retina or the aqueous and the corneal endothelium for example. Like a wave breaking on the shore, energy is dissipated where density changes, and the tissues at these sites are damaged by the shear forces generated. Corneal endothelial loss ${ }^{31}$ and retinal detachment ${ }^{32}$ have been reported in association with YAG capsulotomy. So, unlike the longer pulsed thermal lasers, clinically significant distant mechanical damage from shock and acoustic waves may well be a complicating factor with short pulsed Nd:YAG laser sclerostomies; particularly since the energies used (thirty pulses at $52 \mathrm{~mJ}$ to perforate human sclera in vivo ${ }^{33}$ ) are far in excess of those used for other YAG procedures. Locally, a passage through the tissues is created by three main elements: ${ }^{30}$ plasma formation, disintegrating a small volume of tissue at the laser's focus; plasma expansion, discharging pressure waves disrupting the adjacent tissues; and latent stress, inherent elastic recoil where counteracting three dimensional support is lost. Thus the plasma forming laser blasts its way through the sclera to form a sclerostomy.

Sclerostomies have been formed in rabbits, ${ }^{34}$ primates ${ }^{35}$ and human eyes ${ }^{33.36 .37}$ by March et al. using the Nd: YAG laser delivered through a goniolens. In terms of both cutting accuracy and tissue trauma, performance is poor. Scanning electron microscopic studies of sclerostomies $^{33.36}$ created in human eyes by March et al. show fistula diameters varying between about $300 \mu \mathrm{m}$ and $700 \mu \mathrm{m}$ despite similar laser parameters. The extent of the tissue damage zone about these sclerostomies was not formally examined; but a ragged outline and extensive splitting of the adjacent tissue planes are evident in the cross-sectional views. The internal ostia are surrounded by a wide margin of disruption in Descemet's membrane and the corneal endothelium, with the similar blown out appearance at the external ostia being likened by the authors to that of a small volcano.

Ophthalmic Q-switched Nd:YAG lasers are widely available, but most of the systems currently marketed will not produce peak powers in the regions of those used by March et al. Plasma forming lasers are non-haemostatic and neither clean or accurate in their cutting. Pressure wave damage to distant sites in the eye is likely to be a real hazard at the energy levels required. So there is little to recommend the use of lasers in this category for sclerostomy procedures.

\section{PHOTODISSOCIATIVE (ULTRAVIOLET) LASERS}

Light emitted by the argon/fluorine excimer laser at $193 \mathrm{~nm}$ is strongly absorbed by the organic scleral constituents (as opposed to scleral water for the mid-infrared lasers). The pathlength within which all the incident photons from the excimer laser are absorbed is thus extremely short (less than $5 \mu \mathrm{m}$ ). ${ }^{6}$ In combination with a short pulse width (10-20 nsec), this ensures that a high power density is achieved within a very well circumscribed volume of tissue at the target surface. Importantly though, at fluences below about $500 \mathrm{~mJ} / \mathrm{cm}^{2}$ for the excimer at $193 \mathrm{~nm}$, irradiance is not high enough to induce plasma formation. ${ }^{38}$ At $193 \mathrm{~nm}$ each photon has sufficient energy to break the carbon-carbon bond ubiquitous in organic tissue constituents.

If enough of these bonds are broken simultaneously, a phase transition occurs, and organic molecular fragments are ejected as a gas from the target surface ${ }^{39}$ This is photodissociative ablation. Note that absorbed energy which would otherwise be dissipated thermally, by inducing molecular vibration, is used in breaking carbon-carbon bonds directly. So pure photodissociative ablation is essentially non-thermal. Energy in excess of that required for ablation heats and ejects the gaseous ablation product. But this is expelled before any significant conduction of heat to the adjacent tissues can occur. Marshall et al. ${ }^{40}$ found tissue damage limited to a $300 \mathrm{~nm}$ zone in the walls of rabbit corneal incisions made with the excimer laser at $193 \mathrm{~nm}$. This is almost a thousand times smaller than damage zones about sclerostomies created with thermal lasers. Provided pulse energy is constant, a constant depth of tissue is removed with each pulse. ${ }^{39}$ This has been referred to as the 'etch depth', an analogy that aptly describes the sublime precision with which the excimer laser at $193 \mathrm{~nm}$ cuts through the tissues.

In terms of cutting accuracy and lack of trauma to the adjacent tissues, the performance of the excimer laser at $193 \mathrm{~nm}$ is unsurpassed. Thermal damage is negligible, and shock wave damage is considerably less than that induced by plasma forming lasers. In corneal ablation, endothelial loss attributed to pressure wave damage has been observed; but it was limited to cells directly beneath the line of incision and restricted to a range of $40 \mu \mathrm{m} .{ }^{40}$ 
This should not be significant in the context of sclerostomy formation, since the tissues in the line of fire are all removed.

The excimer laser at $193 \mathrm{~nm}$ has recently been applied by Seiler et al $^{41-43}$ to an ingenious new form of glaucoma surgery in which a $2 \mathrm{~mm}$ by $0.8 \mathrm{~mm}$ block of sclera is ablated away ab externo. Aiming directly at the trabecular meshwork, ablation continues until egress of aqueous automatically halts progress as soon as the canal of Schlemm is entered. The base of this rectangular ablation channel is then dried and ablation proceeds until the canal of Schlemm and the endothelial (high resistance) portion of the trabecular meshwork are removed. This operation is referred to as 'sinusotomy' or 'partial trabeculectomy', and has been performed in humans. ${ }^{41}$ However, it retains the disadvantage inherent in conventional procedures of requiring formal conjunctival dissection (a significant stimulus to local wound healing) prior to laser application, and so could not really be considered as a convenient outpatient procedure.

Light at $193 \mathrm{~nm}$ is not transmitted by the cornea or aqueous, prohibiting gonioscopic delivery, and cannot be passed through an endoscope. Because of these limitations, investigation into sclerostomy procedures has concentrated on the excimer laser at $308 \mathrm{~nm}$, which can be delivered endoscopically. The peak absorption wavelength for scleral organic polymers is $190 \mathrm{~nm} .{ }^{6}$ Hence light at $308 \mathrm{~nm}$ is less well absorbed than at $193 \mathrm{~nm}$, with a corresponding resultant degradation in cutting performance. The longer absorption pathlength at $308 \mathrm{~nm}$ effectively expands the target tissue volume, and a greater irradiance is required to reach the photon density required for photodissociation. Operating fluences and etch depths are thus larger, more photons are transmitted or scattered, and significant thermal damage zones emerge. But these remain relatively small, and results similar to those of the mid-infrared lasers are observed.

Berlin et $a{ }^{44-46}$ using ab interno contact endoscopic delivery of the excimer laser at $308 \mathrm{~nm}$ in rabbits, were able to form sclerostomies with diameters corresponding to endoscopic fibre diameters of $400 \mu \mathrm{m}$ and $600 \mu \mathrm{m}$. Less than 100 pulses at a fluence of $3.5 \mathrm{~J} / \mathrm{cm}^{2}$ were required for perforation. At a repetition rate of $20 \mathrm{~Hz}$ (pulses per second), sclerostomies are completed in four to five seconds. The observed thermal damage zone was less than $100 \mu \mathrm{m}$. This was using a contact delivery system, and considerably smaller damage zones may be possible if this were modified. Fistula patency was maintained for up to four months - an unusually good result in the rabbit model. Muller-Stolzenburg et al. ${ }^{47}$ using a similar technique, also in rabbits, noted a $50 \mu \mathrm{m}$ thermal damage zone and a significant reduction in the IOP of operated compared to control fellow eyes at 60 days. Histological examination revealed that all fistulas were closed by six months.

Ablation is an imprecise term that is, nevertheless, widely used in the literature concerning lasers in ophthalmology. It generally refers simply to the surgical destruc- tion of body tissue. Thus any of the lasers used in sclerostomy formation might be described as 'ablating'. But in this narrow context, ablation has evolved a special connotation: it implies a clear quantitative superiority of cutting performance in terms of both accuracy and lack of trauma to the adjacent tissues. Hence the mid-infrared (photovaporisng) lasers are included in this category alongside the (photodissociative) excimer lasers (Table I); and it is these 'ablating lasers' that have the greatest theoretical potential in laser sclerostomy formation.

\section{DELIVERY SYSTEMS}

Cutting accuracy and tissue trauma are not only influenced by the laser itself. Additional thermal and mechanical damage is produced if hot expanding gases are trapped within the forming sclerostomy by the delivery system (Fig. 2). The mode of delivery also has a major bearing on cutting accuracy, since some methods ensure a more consistent aim than others. Ab interno and $a b$ externo approaches have been described using gonioscopic, endoscopic, and open mask systems. The advantages and disadvantages of these systems are outlined below.

\section{GONIOSCOPIC DELIVERY}

Gonioscopic delivery is possible for lasers at wavelengths transmitted by the cornea and the aqueous. These comprise lasers emitting in the visible, the near infrared, and the near ultraviolet spectra ${ }^{6}(315-1400 \mathrm{~nm})$. Latina et al. using the pulsed dye laser ${ }^{17}$ and March et al. ${ }^{33}$ using the Q-switched Nd:YAG laser have formed sclerostomies $a b$ interno using a modified goniolens. ${ }^{48}$

Gonioscopic delivery is initially attractive for several reasons. First, as with other $a b$ interno methods, conjunctival manipulation is minimal. No dissection is required. The conjunctiva is simply pierced with a fine bore needle, and saline or a viscoelastic agent is injected to balloon the conjunctiva clear of the site of scleral perforation. Avoiding conjunctival dissection removes an important dimension of trauma inherent in conventional glaucoma drainage operations; and, since conjunctival wound leakage is no longer a concern, facilitates the use of pharmacological inhibitors of wound healing such as 5-fluorouracil. Gonioscopic delivery also requires no anterior chamber instrumentation, and gives good access to all four quadrants.

There are, however, some important limitations. Lasers that are not absorbed by the cornea are not well absorbed by the sclera either. So ablating lasers cannot be delivered, and tissue trauma is thus relatively extensive. Cutting accuracy is also suboptimal since consistency of aim is poorly controlled. With reference to the latter, up to 25 consecutive pulses were required by Latina et al. to form a complete sclerostomy in rabbits with the pulsed dye laser, ${ }^{17}$ and 30 pulses were required by March et al. using the Nd:YAG laser in a human eye. The problem of superimposing this number of consecutive pulses accurately through a gonioscope is illustrated by the difference between the histological cross sectional fistula outline 
Table I. A summary of the cutting attributes of lasers used in experimental sclerostomy procedures

\begin{tabular}{|c|c|c|c|c|c|}
\hline & \multirow{2}{*}{$\begin{array}{l}\text { Photodisrupting } \\
\text { lasers }\end{array}$} & \multicolumn{2}{|c|}{ Thermal lasers } & \multicolumn{2}{|c|}{ Ablating lasers } \\
\hline & & Non dye enhanced & Dye enhanced & Mid infrared and $308 \mathrm{~nm}$ & $193 \mathrm{~nm}$ \\
\hline Thermal damage & ++ & +++ & ++ & \pm & - \\
\hline Accuracy & - & \pm & + & +++ & ++++ \\
\hline Cauterising efficiency & - & + & - & - & - \\
\hline
\end{tabular}

obtained by Latina et al. in vitro and in vivo. ${ }^{17}$ Using a fixed laser aimed at a fixed strip of isolated sclera, a smooth cylindrical channel is formed. In vivo, with similar laser parameters and free-hand gonioscopic delivery, a ragged conical outline is produced - the internal ostium being three times larger than the external. Dimensions were inconsistent, with a range of $500 \mu \mathrm{m}$ at the internal and $100 \mu \mathrm{m}$ at the external orifice. Other factors may have been operating; but this is precisely the effect that a drifting aim between pulses might be expected to produce. So it seems reasonable to impute that inaccurate superimposition was mainly responsible.

Results from trials of filtration durability after gonioscopic laser sclerostomy are poor. With Latina's method, using the pulsed dye laser and iontophoretic methylene blue dye enhancement, only two out of six human eyes followed for one year maintained filtration. ${ }^{20} \mathrm{~A}$ comparison of filtration durability in rabbits showed no significant difference with that obtained by conventional thermosclerostomy. ${ }^{18}$ In an earlier summary of results from an uncontrolled series of seventy patients with sclerostomies formed by $\mathrm{March}^{37}$ using a variety of permutations of Q-switched YAG laser, dye enhancement, and a $200 \mu \mathrm{sec}$ pulsed krypton laser; fifty maintained some permanent filtration and lowering of intraocular pressure. These results are difficult to evaluate however, since these patients may have been a heterogeneous group and the period of follow-up was not specified. But again, they compare unfavourably with most trabeculectomy series. ${ }^{27}$

\section{AB INTERNO ENDOSCOPIC DELIVERY}

The problem of superimposing consecutive pulses accurately was highlighted by Gaasterland et al. in $1987 .{ }^{10}$ Their solution was to deliver the laser with a contact endoscope, which is passed across the anterior chamber through a corneal incision, and advanced up the sclerostomy as it forms. Initial placement can be observed through a gonioscope, and completion is marked by the appearance of the endoscopic tip beneath the conjunctiva, which is ballooned clear prior to the procedure as with gonioscopic delivery. Since the probe is fixed at its point of entry through the cornea and by the walls of the forming sclerostomy, a steady aim as the probe advances is ensured.

This method shares with gonioscopic delivery the advantage of minimal conjunctival manipulation, and avoids the problem of superimposition drift. But a corneal incision and instrumentation in the anterior chamber are required. So laser sclerostomy with this mode of delivery, like Seiler's sinusotomy, could not really be thought of as a simple outpatient procedure. Also, access to the opposite quadrant may be limited by a protuberant nose or brow.

Non-contact $a b$ interno delivery has been described. ${ }^{8}$ But this is no more accurate than free-hand gonioscopic delivery and just as invasive as the contact endoscopic approach.

Gaasterland et al. used a continuous wave argon laser. Subsequently others ${ }^{13-15}$ have used contact endoscopic delivery with the continuous wave Nd:YAG laser-also a thermal laser. Unlike gonioscopic delivery, laser selection is not limited by the need for transmission through the cornea and aqueous, and most ablating lasers may be delivered endoscopically. Two of these, the excimer laser at $308 \mathrm{~nm}^{45,47}$ and the Erbium YAG laser ${ }^{26}$ have been used to fashion sclerostomies $a b$ interno in rabbits with encouraging results.

However, although some of the more accurate lasers may be used, their potential may not be fully realised using contact endoscopic techniques. Hot ablation products, normally ejected rapidly from the target surface, are contained by the presence of the endoscope blocking their exit from the sclerostomy (Fig. 2). This increases both mechanical and thermal damage. Berlin, using the excimer laser at $308 \mathrm{~nm}$ in rabbits, observed side channels about the sclerostomy. He interpreted these as being splits in planes of tissue cleavage formed by containment of the expanding gases. ${ }^{46}$ Using the Erbium:YAG laser in isolated human sclera, ${ }^{22}$ Berlin also demonstrated thermal damage zones for contact endoscopic delivery twice the size of those for non-contact techniques. Presumably trapping of hot gaseous ablation products within the forming sclerostomy results in additional conductive thermal damage (Fig. 2).

\section{AB EXTERNO ENDOSCOPIC DELIVERY}

To avoid conjunctival dissection, research has until recently focussed on $a b$ interno delivery. Ab interno endoscopic techniques are more accurate than gonioscopic methods, but are relatively invasive. As a compromise, a 'hockey stick' endoscopic probe has been developed. This delivers the laser at almost $90^{\circ}$ to the line of the shaft, and can be passed through a $1 \mathrm{~mm}$ conjunctival incision to be positioned over the site of ablation. Conjunctival dissection remains minimal, and instrumentation in the anterior chamber is avoided. There is reduced danger of inadvertent conjunctival perforation at the sclerostomy site; and, provided that the laser used is strongly absorbed by aqueous (this is true of all the ablating lasers), there is no danger to the intraocular structures once the sclerostomy is complete. 
Human trials using this system to deliver the holmium:YAG laser are currently in progress. ${ }^{21}$ Early results are again disappointing, with almost half the eyes failing to maintain filtration at six months. ${ }^{21}$ The sclerostomies were relatively fine bore $(100-250 \mu \mathrm{m})$ however, and this was a contact endoscopic technique with no provision for exhaust of ablation effluent. So the tissue damage zone may have been larger than expected. There may be a critical relationship between fistula size and the size of the surrounding tissue damage zone governing the tendency for closure through wound healing. Certainly, the evidence from rabbit experimentation would seem to suggest that reducing the zone of thermal damage prolongs fistula survival. $^{8,26,44}$ Thermal damage zones of up to $140 \mu \mathrm{m}$ have been observed with unmodified contact delivery of the similar erbium:YAG laser. ${ }^{22}$ Damage zones around onetenth this size are possible ${ }^{24}$ using mid infrared lasers; but these results will only be reproduced in vivo if secondary thermal and mechanical damage from trapped ablation products is avoided.

Eye movements during the procedure or variation in the tilt of the operator's hand do allow for some deviation of aim between pulses with the hockey stick technique; and the angle at which the sclerostomy enters the anterior chamber is not well controlled. But the holmium laser only takes about four seconds to fashion a sclerostomy, allowing little time for aim to drift; and the angle at which a sclerostomy enters the anterior chamber should not be critical, provided that the internal ostium is sufficiently clear of the iris plane to minimise the likelihood of its being blocked by prolapsing iris if the anterior chamber should shallow postoperatively.

The ability to place the internal ostium in a relatively high position above the iris plane is an important feature of $a b$ externo delivery methods. Passing an endoscope through the anterior chamber requires entry through the peripheral cornea; whereas external delivery may aim up towards the axial cornea, clearing the iris plane by a greater margin. Additional procedures, such as a peripheral iridectomy to discourage AC shallowing or initial photocoagulation of the iris base to discourage iris prolapse, are thus more likely to be required with $a b$ interno than ab externo approaches.

\section{OPEN MASK DELIVERY AB EXTERNO}

The excimer laser at $193 \mathrm{~nm}$ cuts more accurately than the other ablating lasers with almost no trauma to the adjacent tissues, but has been considered impractical in the context of sclerostomy formation because it cannot be delivered gonioscopically or endoscopically. To overcome these limitations, an open mask system has been designed, and is the subject of current experimental evaluation.

An open mask is simply a shield of material the laser will not ablate with a central aperture. This primary aperture defines the sclerostomy outline, whilst the surrounding tissues are shielded from the incident beam. The mask is positioned by alignment with the limbus, and the angle of entry into the anterior chamber is controlled by a secondary aperture through which the laser is lined up with the primary aperture. Small eye movements are tolerated so long as the beam still embraces the primary aperture.

Movement of the mask relative to the eye, and hence the problem of superimposition drift, is avoided by vacuum fixation on the cornea. Before this, the conjunctiva is also fixed by gentle suction beneath the main portion of the mask, and hitched forward, or plicated at the limbus in such a way that once the mask is removed at the end of the procedure the conjunctival wound falls back clear of the sclerostomy. Again, formal dissection of the conjunctiva is avoided.

In contrast with contact endoscopic techniques, including the unmodified $a b$ externo 'hockey stick' system, secondary thermal and mechanical damage from trapped ablation effluent does not occur. So the potential accuracy of the ablating lasers may be fully utilised with an open mask technique. Although an exhaust mechanism could easily be accommodated in the hockey stick system, this would probably increase the bore of the instrument, requiring a larger conjunctival incision.

The main disadvantage of ablating lasers in comparison with thermal lasers producing a large zone of adjacent tissue coagulation is that cutting is non-haemostatic. An airjet directed en-face into the forming microsclerotomy can be built into an open mask system to raise the ambient pressure in the target area. This tamponades any transected small vessels, maintaining haemostasis during ablation.

Some features of delivery systems used in laser sclerostomy formation are summarised in Table II.

\section{DISCUSSION}

The best results in terms of consistent accuracy of fistula size and minimising the adjacent tissue damage zone should be obtained with an ablating laser delivered either through an open mask or a modified endoscopic system incorporating an efficient exhaust mechanism. The excimer laser at $193 \mathrm{~nm}$ cuts with greater precision than the other ablating lasers but is relatively slow in comparison with the mid-infrared lasers. Which of these lasers

Table II. A summary of the attributes of delivery systems used in experimental laser sclerostomy procedures

\begin{tabular}{|c|c|c|c|c|}
\hline & \multicolumn{2}{|c|}{ Ab externo } & \multicolumn{2}{|c|}{ Ab externo } \\
\hline & Gonioscopic & Endoscopic & Endoscopic & Open mask \\
\hline Conjunctival dissection & Injection only & Injection only & $1 \mathrm{~mm}$ linear incision & Defect $<$ diam. sclerostomy \\
\hline Access to quadrants & 4 & $2-3$ & $3-4$ & $2-3$ \\
\hline Pulse to pulse superimposition accuracy & Poor & Good & Fair & Good \\
\hline Compatibility with ablating lasers & No & Yes (not $193 \mathrm{~nm}$ ) & Yes (not 193 nm) & Yes (incl. 193 nm) \\
\hline Trapping of gaseous product & Yes & Yes & Yes & No \\
\hline
\end{tabular}


predominates eventually will depend on whether the near perfect sclerostomies formed by the excimer laser at $193 \mathrm{~nm}$ are shown to be significantly more durable in vivo. It may be that older patients, who might be less tolerant of a slightly longer procedure, would be better suited to the faster ablating lasers; whereas younger patients, with their more aggressive wound healing, would fair better with the excimer laser at $193 \mathrm{~nm}$.

Laser sclerostomy is an unguarded procedure. Conventional fully penetrating drainage procedures such as thermosclerostomy or trephination have been largely abandoned in favour of trabeculectomy because they are more prone to the perioperative complications associated with a flat anterior chamber, and subsequent formation of a thin cystic drainage bleb with the attendant risk of late endophthalmitis. It seems that the ideal drainage fistula imparts a degree of resistance to outflow sufficient to prevent these complications. Trabeculectomy provides this resistance; but inconsistently, since it relies on subjectively controlled factors such as suture tension in scleral flap apposition.

Seiler's technique of partial trabeculectomy (sinusotomy) using the excimer laser at $193 \mathrm{~nm}$ may improve upon this, but this is by no means certain. Following this procedure in human cadaver eyes, ${ }^{41}$ the perfusion rate required to maintain a set pressure in each of these eyes shows some variation $(0.713-1.778 \mu \mathrm{m} / \mathrm{min})$ despite constant laser parameters, implying a degree of variability in outflow resistance.

With an ablating laser and a delivery system that does not subtract from cutting performance, it may be easier to reproduce consistent fistula dimensions in laser sclerostomy than the tension of flap apposition in trabeculectomy or ablation depth in sinusotomy. Outflow resistance might then be controlled within an acceptable range by a scaled down sclerostomy, or 'microsclerostomy', small enough to impart sufficient resistance to aqueous flow to avoid the undesirable attributes of larger bore fully penetrating fistulas, but large enough to resist closure through wound healing.

If this balance can be reliably found in a convenient, uncomplicated outpatient procedure, laser microsclerostomy may open the door to primary surgical treatment in all newly diagnosed cases of primary open angle glaucoma.

Key words: Filtration Glaucoma Laser Sclerostomy Surgery.

\section{REFERENCES}

1. Mao LK, Stewart WC, Shields MB: Correlation between intraocular pressure control and progressive glaucomatous damage in primary open angle glaucoma. Am J Ophthalmol 1991, 111: 51-5.

2. Yildirim E, Bilge AH, Ilker S: Improvement of visual field following trabeculectomy for open angle glaucoma. Eye 1990, 4: 103-6.

3. Funk $J$ and Grehn $F$ : Changes in the temporal neuroretinal border area of the papilla following pressure reducing surgery. Fortschr Ophthalmol 1989, 86: 727-31.

4. Jay JL and Allan D: The benefit of early trabeculectomy ver- sus conventional management in primary open angle glaucoma relative to severity of disease. Eye 1989, 3: 528-35.

5. Lavin MJ, Wormwald RL, Migdal CS, Hitchins RA: The influence of prior therapy on the success of trabeculectomy. Arch Ophthalmol 1990, 108: 1543-8.

6. Marshall J: Lasers in ophthalmology: the basic principles. Eye 1988, 2 (Suppl): 98-112.

7. Beckman H, Rota A, Barraco R, et al.: Limbectomies, keratectomies, and keratostomies performed with a rapid pulsed carbon dioxide laser. Am J Ophthalmol 1971, 71: 1277-83.

8. Jaffe GJ, Williams GA, Meiler WF, Radius RL. Ab interno sclerostomy with a high-powered argon endolaser. Am J Ophthalmol 1988, 106: 391-6.

9. Jaffe GL, Williams GA, Meiler WF, Radius RL, et al.: Ab interno sclerostomy with a high-powered argon endolaser clincopathalogic correlation. Arch Ophthalmol 1989, 107: 1183-5.

10. Gaasterland DE, Hennings DR, Boutacoff TA, Bilek C: Ab interno and ab externo filtering operations by laser contact surgery. Ophthalmic Surg 1987, 18: 254-7.

11. Shimokaji G and Stamper RL: Argon laser sclerostomy compared with trabeculectomy. Invest Ophthalmol Vis Sci 1991, 32 (ARVO Suppl): 936.

12. Odrich SA, Eaton AM, Schulbert H: Fluorescein dye enhanced argon laser sclerostomy. Invest Ophthalmol Vis Sci 1991, 32 (ARVO Suppl): 934.

13. Higginbotham EJ, Kao G, Peyman G: Internal sclerostomy with the Nd:YAG contact laser versus thermal sclerostomy in rabbits. Ophthalmology 1988; 95: 385-90.

14. Javitt JC, O'Connor SS, Wilson RP, Federman JL: Laser sclerostomy ab interno using a continuous wave Nd:YAG laser. Opthalmic Surg 1989, 20: 552-6.

15. Federman JL, Wilson RP, Ando F, Peyman GA: Contact laser thermal sclerostomy ab interno. Ophthalmic Surg 1987, 18: 726-7.

16. Latina MA, Goode S, deKater AW et al.: Experimental sclerostomies using a pulsed dye laser. Lasers Surg Med 1988, 8: 233-40.

17. Latina MA, Dobrogowski MD, March WF, Birngruber R: Laser sclerostomy by pulsed dye laser and goniolens. Arch Ophthalmol 1990, 108: 1745-50.

18. Rabowsky JH, Dukes AJ, Lee DA, Panek WC: Pulsed dye laser internal sclerostomy vs filtration surgery in a rabbit model. Invest Ophthalmol Vis Sci 1991, 32 (ARVO Suppl): 387.

19. Melamed S, Solomon A, Neumann D, et al.: Internal sclerostomy using laser ablation of dye enhanced sclera in glaucoma patients: a pilot study. Invest Ophthalmol Vis Sci 1991, 32 (ARVO Suppl): 935.

20. Latina MA and March WF: Ab interno sclerostomy using a goniolens and pulsed dye laser in glaucoma patients: a preliminary study. Invest Ophthalmol Vis Sci 1991, 32 (ARVO Suppl): 938.

21. Hoskins HD, Iwach AG, Vassiliadis A et al:: Subconjunctival THC:YAG laser thermal sclerostomy. Ophthalmology 1991, 98: 1394-1400.

22. Berlin M, Martinez M, Peter-Loercher H: Erbium YAG laser sclerstomy, mechanism and histological analysis. Invest Ophthalmol Vis Sci 1991, 32 (ARVO Suppl): 939.

23. Farnath D, Margolis TI, Puliafito CA: Mid infrared laser sclerostomy. Lasers Light Ophthalmol 1988, 2: 196.

24. Margolis TI, Farnath DA, Puliafito CA: Mid infrared laser sclerostomy. Invest Ophthamol Vis Sci 1988, 29 (ARVO Suppl): 366.

25. Eaton AM, Odrich MD, Hermann D, et al.: Holmium and Thulium laser sclerostomy a comparative study. Invest Ophthalmol Vis Sci 1991, 32 (ARVO Suppl): 940.

26. Hill RA, Ozler SA, Baerveldt $\mathrm{J}$, et al.: Ab interno neodymium: YAG versus erbium: YAG laser sclerostomies in 
rabbits. Invest Ophthalmol Vis Sci 1991, 32 (ARVO Suppl): 388.

27. Skuta GL and Parrish RK: Wound healing in glaucoma filtering surgery. Surv Ophthalmol 1987, 32: 149-70.

28. L'Esperance FA Jr: Ophthalmic lasers: photocoagulation, photoradiation and surgery. 2nd ed. St Louis, Mosby Co. 1983, 538-543.

29. March WF, Shaver RP, Gherezghiher T, Nordquist RE: Silver oxide in YAG sclerostomy. Lasers Surg Med 1987, 7: $353-4$.

30. Mainster MA, Sliney DH, Belcher DC, Buzney MS: Laser photodisrupters: damage mechanisms, instrument design and safety. Ophthalmology 1983, 90: 973-91.

31. Khodadoust AA, Arkfeld DF, Caprioli J, Sears ML: Ocular effects of neodymium-YAG laser. Am J Ophthalmol 1984, 98: $144-52$.

32. Terry AC, Stark WJ, Maumanee AE: Anterior segment surgery Baltimore, Wilkins and Wilkins 1987: 383-387.

33. March WF, Koss MC, Gherezghiher T, et al.: Histologic study of a neodymium-YAG laser sclerostomy. Arch Ophthalmol 1985, 103: 860-3.

34. March WF, Bernitsky D, Gherezghiher T, et al:: Creation of filtering blebs with the YAG laser in primates and rabbits. Glaucoma 1985, 7: 43-5.

35. Gherezghiher T, March WF, Koss MC, et al.: Neodymium: YAG laser sclerostomy in primates. Arch Ophthalmol 1985, 103: $1543-5$.

36. March WF, Gherezghiher T, Koss MC, et al.: Experimental YAG laser sclerostomy. Arch Ophthalmol 1984, 102: 1834-6.

37. March WF: Long-term follow-up of patients undergoing laser sclerostomy. Ophthalmic Laser Ther 1987, 2: 161-5.

38. van Saarloos PP and Constable IJ: Bovine corneal stroma ablation rate with $193 \mathrm{~nm}$ excimer laser radiation: quantitative measurement. Refract Corneal Surg 1990, 6: 424-9.

39. Dyer PE and Srinivasen R: Nanosecond photoacoustic studies on ultraviolet laser ablation of organic polymers. Applied Physics Letters 1986, 48: 445-7.

40. Marshall J, Trokel S, Rothery S, Kruger RR: A comparative study of corneal incisions induced by diamond and steel knives and two ultraviolet radiations from an excimer laser. Br J Ophthalmol 1986, 70: 482-501.

41. Seiler T, Kriegerowski M, Bende T, Wollensak J: Partielle externe trabekulektomie. Klin Mbl Augenheilk 1989, 195: 216-20.

42. Seiler T, Kriegerowski M, Bende T: Partial external trabeculectomy (PET). Lasers Light Ophthalmol 1989, 2: 196.

43. Seiler T, Kriegerowski M, Bende T, Wollensak J: Partial trabeculectomy with the excimer laser $(193 \mathrm{~nm})$. Invest Ophthalmol Vis Sci 1988, 29 (ARVO Suppl): 239.

44. Berlin MS, Rajacich G, Duffy M, et al.: Excimer laser photoablation in glaucoma filtering surgery. Am J Ophthalmol 1984, 103: 713-14.

45. Berlin MS, Martinez M, Papaioannou T, Grundfest W: Goniophotoablation: excimer laser glaucoma filtering surgery. Lasers Light Ophthalmol 1988, 2: 17-24.

46. Berlin M: Excimer laser sclerostomy. Auto-Digest Ophthalmol 1991, 29: 1.

47. Muller-Stolzenburg N, von Haebler M, Erb C, Buchwald $\mathrm{HJ}$ : $\mathrm{Ab}$ interno excimer laser sclerostomy in ablino rabbits-influence of a topically applied UV absorber. Invest Ophthalmol Vis Sci 1991, 32 (ARVO Suppl): 941.

48. March WF, LaFuenk H, Rol P: Improved goniolens for YAG sclerostomy. Ophthalmic Surg 1988, 19: 513. 